\title{
Should Labor be Routinely Induced from 39 Weeks of Gestation?
}

\author{
Daniel Faustin* \\ Maternal Fetal Medicine, Wyckoff Heights Medical Center, USA
}

*Corresponding author: Daniel Faustin, Maternal Fetal Medicine, Wyckoff Heights Medical Center, Brooklyn, NY, USA.
Received Date: July 10, 2018

Published Date: July 13, 2018

\section{Editorial}

Extended delay in the onset of spontaneous labor at term continues to be a concern of practicing obstetricians due to the potential neonatal complications of post-maturity. The perinatal mortality rate is known to progressively increase from 40 weeks of gestation in otherwise uncomplicated pregnancies [1,2], but there have been conflicting reports regarding whether routine induction of labor would be associated with increased likelihood of primary c/section. One recent study addresses these issues and provides statistical evidence for the maternal benefits of elective induction of labor at 39 weeks of gestation without compromising fetal safety.

The report by Grobman in the American Journal of Obstetrics and Gynecology was about an NIH study comparing induction of labor (IOL) at 39 weeks of gestation with expectant management (EM) among low-risk nulliparous women [3]. Although no significant difference was seen in the frequency of adverse perinatal outcomes, a lower frequency of caesarean deliveries was observed in the induction of labor group (18.6\%) compared to the expectant management group (22.2\%), as well as a lower incidence of preeclampsia/gestational hypertension (9.1 vs 14.1 respectively). The patient population comprised 6106 women randomized at 41 hospitals: 3062 in the induction of labor group and 3044 in the expectant management group.
One of the limitations of applying this plan of care to the majority of pregnancies reaching term is the accurate determination of the gestational age. Another hurdle to achieving successful active labor followed by vaginal delivery is the unripe cervix. These two factors can be circumvented with the institution of early prenatal care with confirmatory ultrasonographic determination of gestational age and the judicious use of both medical and mechanical methods of cervical ripening prior to the initiation of labor induction.

For complicated pregnancies with increased likelihood of adverse maternal or fetal outcome, the timing and mode of delivery are dictated by the nature of the clinical entity. However when normal pregnancy reaches $390 / 7$ weeks and the conditions are met for safe induction of labor, the benefits of delivery to the pregnant woman and her offspring seem to outweigh any theoretical risk.

\section{References}

1. Hollis B (2002) Prolonged Pregnancy. Curr Opin Obstet Gynecol 14(2): 203-207.

2. Rad L, Robinson JN, Economy KE, Norwitz ER (2000) Postterm Induction of Labor Revisited. Obstet Gynecol 96(5 pt 1): 779-783.

3. William Grobman (2018) LB01: A randomized trial of elective induction of labor at 39 weeks compared with expectant management of low-risk nulliparous women. Am J Obstet Gynecol 218(1): S601. 